\title{
Une forme caractéristique de condition de l'étude dans la réalisation d'une ingénierie didactique en mathématiques à l'école primaire
}

Describing the joint action of the teacher and the students in a math lesson

Serge Quilio

\section{OpenEdition}

\section{Journals}

Édition électronique

URL : http://journals.openedition.org/educationdidactique/1470

DOI : 10.4000/educationdidactique. 1470

ISSN : 2111-4838

Éditeur

Presses universitaires de Rennes

\section{Édition imprimée}

Date de publication : 30 octobre 2012

Pagination : $9-26$

ISBN : 978-2-7535-1984-8

ISSN : 1956-3485

\section{Référence électronique}

Serge Quilio, «Une forme caractéristique de condition de l'étude dans la réalisation d'une ingénierie didactique en mathématiques à l'école primaire », Éducation et didactique [En ligne], 6-2 I octobre 2012 mis en ligne le 30 octobre 2014, consulté le 10 décembre 2020. URL : http://journals.openedition.org/ educationdidactique/1470; DOI : https://doi.org/10.4000/educationdidactique.1470 


\title{
UNE FORME CARACTÉRISTIQUE DE CONDITION DE L'ÉTUDE DANS LA RÉALISATION D'UNE INGÉNIERIE DIDACTIQUE EN MATHÉMATIQUES À L'ECOLE PRIMAIRE
}

Serge Quilio (ENS Lyon - IFÉ, ADEF)

\begin{abstract}
Résumé: Cet article développe une perspective d'analyse de leçons de mathématiques dans le cadre de la théorie de l'action conjointe en didactique. Nous montrons l'intérêt de considérer l'action du professeur dans la réalisation effective de ces leçons au sein de transactions didactiques que nous décrivons en terme de jeux. Nous posons ces derniers comme des modèles de description de l'action conjointe du professeur et des élèves. Les épisodes analysés selon cette perspective montrent ainsi une modalité caractéristique de la direction de l'étude des élèves par le professeur. Nous désignons par les termes « temps des situations » le mode de transposition didactique que le professeur doit réaliser pour faire fonctionner la situation observée.
\end{abstract}

Mots clés: jeux d'apprentissage, transaction didactique, action conjointe, professeur, mathématiques

\section{Introduction}

Les éléments que je présente dans cet article sont constitués dans un cadre bien particulier puisqu'ils sont le fruit d'observations effectuées à partir de films vidéos tournés au Centre pour l'Observation et la Recherche de l'Enseignement des Mathématiques de Talence, plus familièrement connu sous la dénomination COREM dans le champ de la didactique des mathématiques. Ce centre pour l'observation des phénomènes didactiques en mathématiques a été créé en 1971 et a fonctionné jusqu'en 1994, date du départ de son fondateur Guy Brousseau, titulaire du premier prix Félix Klein attribué par l'« International Commission on Mathematical Instructions » (ICMI) à un chercheur en mathématiques pour ses travaux sur l'enseignement.

Les épisodes analysés dans ce qui suit sont issus d'une leçon de mathématiques extraite de Rationnels et Décimaux dans la scolarité obligatoire (Brousseau \& Brousseau, 1987) qui est une ingénierie de grande envergure conçue pour l'apprentissage des nombres rationnels et décimaux au CM2 de l'école primaire. Ce curriculum n'est pas une ingénierie modèle et innovante destinée à devenir une nouvelle méthode d'enseignement. C'est une tentative de production de connaissances mathématiques selon un processus qui simulerait une genèse de concepts par une activité ressemblant à celle des mathématiciens: « Les genèses artificielles que nous envisageons de construire devront faire fonctionner la notion de décimal de façon à simuler les différents aspects actuels du concept. Il ne s'agit pas de reproduire le processus historique mais produire des effets similaires par d'autres moyens. La phénoménotechnique épistémologique consiste à faire, sur certains points, des choix très différents que ceux que suggérait l'histoire, et de restaurer, par l'exercice des règles et des principes que l'on a pu découvrir, un processus néanmoins équivalent » (ibid. p. 456).

Ces leçons ont déjà fait l'objet d'analyses didactiques (cf. notamment Brousseau, 1981; Centeno, 1995; Ratsimba-Rajohn, 1992) centrées en quelque sorte sur les savoirs dans la mesure où ce sont les statuts ou le fonctionnement des savoirs qui y ont été étudiés. Il s'agit dans ce qui suit de revenir sur une de ces leçons filmées lors de l'expérimentation et de focaliser la description sur ce que fait un professeur pour faire vivre la situation en utilisant les outils théoriques de l'action conjointe en didactique (Sensevy \& Mercier, 2007; Sensevy, 2011). Ce faisant, nous allons envisager le processus de diffusion du savoir mathématique tout en gardant au cour de ce travail la focalisation sur les contenus de savoir, notamment en centrant l'attention sur l'usage, par le professeur, des ressources du langage, dans l'orientation du travail des élèves. Je propose avant cela de revenir sur l'ensemble du curriculum afin de mieux comprendre le contexte de la leçon analysée. 


\section{Le contexte de l'analyse présenté et la question posée}

L'ensemble des leçons de "Rationnels et décimaux dans la scolarité obligatoire " a été régulièrement mis en œuvre par les enseignants de l'école primaire Jules Michelet de Talence (France) avec des élèves de 10-11 ans (quatrième et cinquième primaires) à partir des années 1970, dans le cadre des activités scientifiques conduites au COREM.

Ce processus d'enseignement n'a pas été conçu pour être utilisé par les enseignants sans une formation et une assistance didactique solides. L'intention de Guy Brousseau n'était pas de proposer un modèle d'enseignement mais de montrer qu'il est possible d'enseigner les décimaux à des élèves " ordinaires ", à l'aide de situations où les connaissances sont construites avec rigueur par les élèves eux-mêmes. Cet objectif a permis à Brousseau de produire des connaissances théoriques essentielles dans le champ de la didactique des mathématiques.

Soulignons que ce mode d'enseignement demande du temps non seulement pour appréhender avec les élèves les problèmes qui s'y posent, mais aussi pour faire fructifier les concepts introduits. Ainsi le processus comprend soixante-cinq leçons se déroulant sur l'intégralité d'une année scolaire, et toutes les notions mathématiques relatives aux rationnels et aux décimaux y sont abordées dans ce que Brousseau appelle "une série génétique de situations ».

Cette série est organisée en deux phases distinctes: l'enseignement des fractions/mesures y précède celui des fractions/applications. Le problème initial de la série conduit à l'invention, par les élèves, d'un moyen de désigner des grandeurs très inférieures à l'unité. Par exemple, l'épaisseur d'une feuille de papier, la masse d'un très petit clou, la contenance d'un dé à coudre de poupée. On demande ainsi aux élèves, qui ont l'expérience des questions de mesure des grandeurs depuis plus d'un an (Brousseau, 1987) et qui ont utilisé une « règle pour mesurer des épaisseurs » (un pied à coulisse), de « trouver un moyen pour désigner cette épaisseur de manière à distinguer entre elles cinq types de feuilles, d'épaisseur différente».

Il s'agit de produire une notation et ses usages pour rendre compte d'une pratique générique, la mesure, dans un cas spécifique où l'opération semble impossible. Pour obtenir une épaisseur mesurable les élèves devront mettre ensemble plusieurs feuilles identiques et ils devront désigner « le papier de tel type » par le fait que « 13 feuilles de ce papier ça fait deux millimètres », ce qui deviendra la fraction 2/13 mm. Ainsi 2/13 désigne l'épaisseur d'une feuille de papier « telle que si on en rassemble 13 semblables le paquet mesurera 2 millimètres $»$.

Avant d'apparaître comme des objets mathématiques reconnaissables, des fractions, les désignations inventées par les élèves seront donc mises à l'épreuve de situations de classement, d'addition, etc. Un grand nombre de leçons sera nécessaire à la construction de l'ensemble des nombres rationnels puis, lorsque les élèves chercheront un ensemble de nombres au maniement plus aisé, à la construction de l'ensemble des fractions décimales et à la notation décimale de position qui s'ensuit.

Vient alors, après 35 leçons la deuxième phase de la série, où il est demandé aux élèves d'agrandir des objets matériels à partir de l'indication de mesures entières, puis fractionnaires, et enfin décimales. Ils doivent en fait étudier des applications linéaires correspondant à des agrandissements où des réductions de figures. Ils devront donc dissocier dans les situations ce qui correspond à des grandeurs (mesures) et ce qui correspond à des scalaires (les opérateurs). Ils étudieront ainsi l'usage et le sens de la multiplication ou de la division par un rationnel ou un décimal.

Nous n'irons pas plus loin dans la description de l'ingénierie ${ }^{1}$ car nous voulons rendre compte du passage à cette deuxième phase, en observant une leçon où les élèves doivent trouver les mesures d'une figure à reproduire à partir de mesures proposées par le professeur et exprimées sous la forme d'une fraction. Nous allons observer comment le professeur observé réalise ce passage en introduisant notre travail par la question suivante: "Sous quel mode un professeur réalise-t-il ce processus et peut-il faire fonctionner la situation auprès des élèves? »

\section{Le cadre théorique des analyses}

Répondre à cette question suppose non seulement que l'on puisse décrire le savoir mathématique 
qui fait l'enjeu de la relation, mais encore que l'on sache décrire les relations didactiques à l'œuvre dans la situation réalisée. Dans la théorie de l'action conjointe en didactique, désormais TACD (Sensevy $\&$ Mercier, 2007; Sensevy, 2011) cette question est abordée en considérant que l'action du professeur et des élèves est conjointe, c'est-à-dire que l'action est toujours sous le regard d'un autre et donc, qu'elle lui est adressée: elle est transaction au sens de Vernant (1997). Ainsi pour nous et à la suite de ces auteurs, l'action dans un milieu symbolique ou matériel a une dimension sémiotique, elle fait signe.

J'emprunterai aussi à Sensevy $(2007,2011)$ la notion de « jeu d'apprentissage » afin de comprendre l'organisation des transactions didactique in situ (Quilio, 2008) et de leur dimension discursive. Cela permet de produire à la fois une description dans la sémantique d'un jeu (les transactions didactiques sont vues ${ }^{2}$ comme si le professeur et les élèves jouaient à un jeu) et aussi une forme d'explication (Wittgenstein, 2005). La notion de « jeu » permet d'utiliser les notions théoriques usuelles dans l'analyse de l'action didactique: contrat didactique, milieu, régulations et dévolutions successives et de travailler sur les descripteurs de l'action conjointe, la topogenèse (comment: qui agit), la mésogenèse (comment: quoi est agi), la chronogenèse (comment: quand l'action), en les articulant grâce au modèle que fournit la notion de jeu coopératif. La notion de jeu d'apprentissage constitue ainsi un outil pour décrire le jeu du professeur sur le jeu de l'élève, les catégories précitées (en particulier celles de milieu et de contrat) jouant un rôle descriptif et conceptuel majeur.

\section{Le cadre méthodologique}

La mise en évidence des jeux qui organisent l'action conjointe est réalisée à partir des interactions langagières entre l'enseignant et les élèves.
$\mathrm{Au}$ sein de cet article, les interlocutions sont relevées dans les transcriptions d'une séance réalisée en 1989 pour la mise en œuvre de l'ingénierie. Chaque intervenant a été précisément identifié, et la transcription rend compte d'un enregistrement vidéo original, au COREM. Cette vidéo a été reprise pour l'analyse des données langagières, une analyse qui s'appuie sur les traces de l'organisation de l'action contenues dans les interventions successives (Sensevy \& Mercier, 2007).

Dans ce travail d'analyse, la constitution d'un synopsis d'action est centrale (Sensevy, 2011). Nous procédons ainsi à un premier résumé de l'action filmée sous la forme d'un tableau synoptique. Ce travail de réduction et de reconstitution de la pratique, à partir des éléments de savoir en jeu dans les transactions, permet de constituer des unités de découpage tout en conservant une forme d'unité de la pratique. Le tableau synoptique permet de localiser des épisodes potentiellement pertinents pour l'élucidation de l'action conjointe et d'envisager la mise en relation d'unités temporellement disjointes dans le déroulement de l'action effective.

\section{Le travail de description}

L'intrigue de la leçon est décrite à partir des jeux d'apprentissage qui structurent, dans la modélisation utilisée ici, les transactions didactiques. On notera l'empreinte des conditions expérimentales de cette leçon, puisque l'intervention du chercheur/concepteur de l'ingénierie a été conservée. On soulignera la durée particulièrement longue de la leçon, 1 heure vingt minutes, qui se déroule dans une classe de l'école primaire: elle peut signifier la difficulté de l'exercice, pour le professeur et les élèves.

Le déroulement de l'intégralité de la séance est résumé dans le tableau synoptique suivant ${ }^{3}$ : 
UNE FORME CARACTÉRISTIQUE DE CONDITION DE L'ÉTUDE ...

Serge Quilio

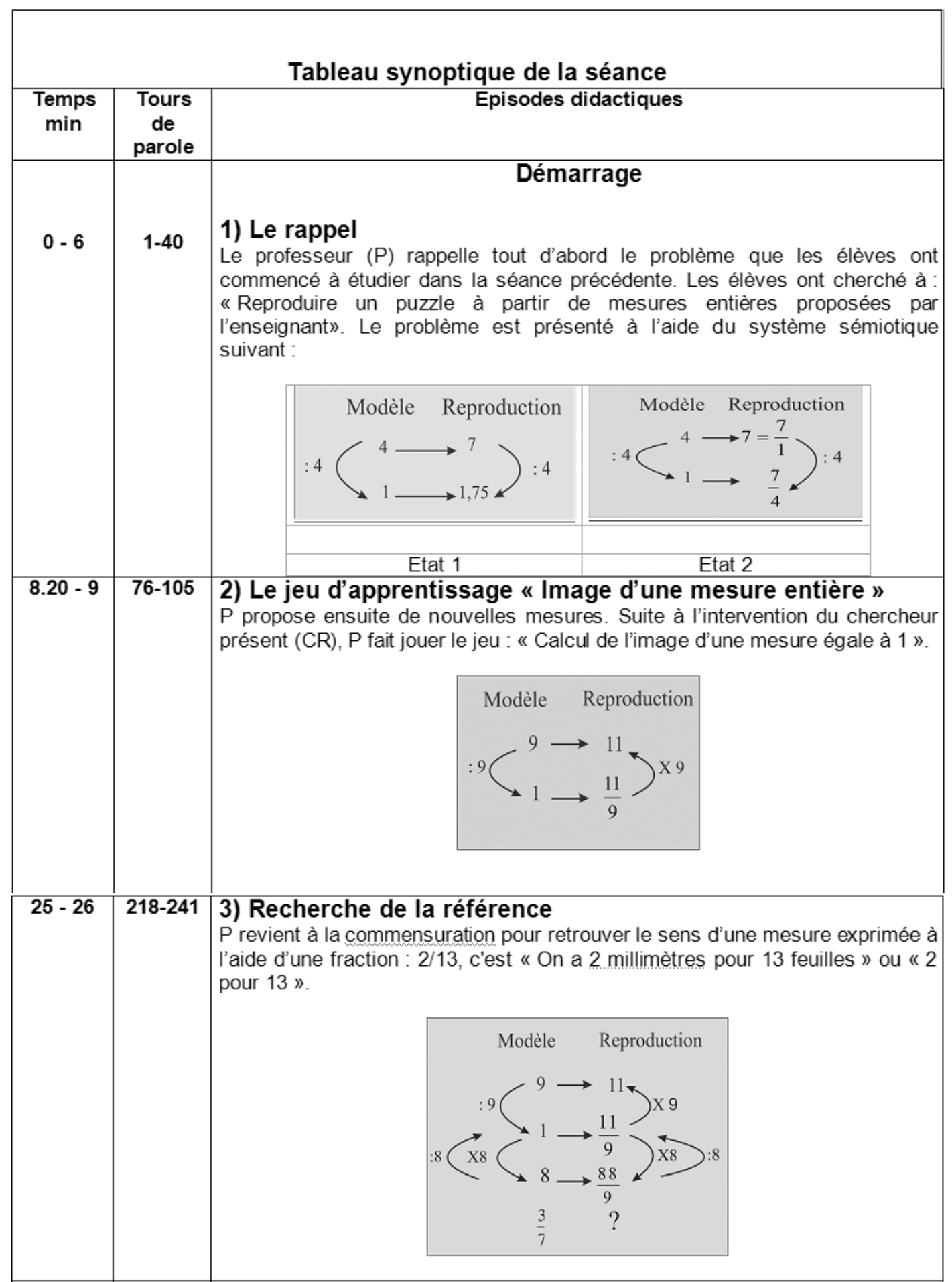




\begin{tabular}{|c|c|c|}
\hline & & $\begin{array}{l}\text { 4) Le jeu d'apprentissage " Image d'une mesure exprimée en } \\
\text { fraction " } \\
\text { P propose ensuite de trouver l'image de la mesure } 3 / 7 \text {. II fait jouer le jeu } \\
\text { "Image d'une mesure exprimée sous la forme d'une fraction ». Pas de } \\
\text { stratégie disponible. }\end{array}$ \\
\hline $\begin{array}{l}44.30- \\
44.45\end{array}$ & 313-359 & $\begin{array}{l}\text { 5) Proposition de I'intermédiaire } 1 / 7 \\
\text { P propose de passer par l'intermédiaire de } 1 / 7 \text { pour trouver l'image de } 3 / 7 \text {. } \\
\qquad \begin{array}{cc|}\text { Modèle } & \text { Reproduction } \\
9 & 11 \\
\frac{7}{7}=\frac{1}{1} & \frac{11}{9} \\
\frac{3}{7} & ? \\
\frac{1}{7} \longrightarrow \\
\end{array}\end{array}$ \\
\hline $\begin{array}{l}56.30- \\
60.30\end{array}$ & $432-468$ & $\begin{array}{l}\text { 6) Le jeu « Transformation d'une mesure décimale en mesure } \\
\text { fractionnaire " } \\
P \text { fait jouer le jeu: "Transformation d'une mesure décimale en mesure } \\
\text { fractionnaire " pour trouver l'image de } 3 / 7\end{array}$ \\
\hline \multirow[t]{2}{*}{$\begin{array}{c}66.45- \\
69\end{array}$} & $514-566$ & $\begin{array}{l}\text { 7) Proposition de l'intermédiaire } 3 \\
\text { Poriente les élèves vers } 3 \text { qui est l'autre intermédiaire permettant de trouver de } \\
\text { l'image de } 3 / 7 \text {. }\end{array}$ \\
\hline & & 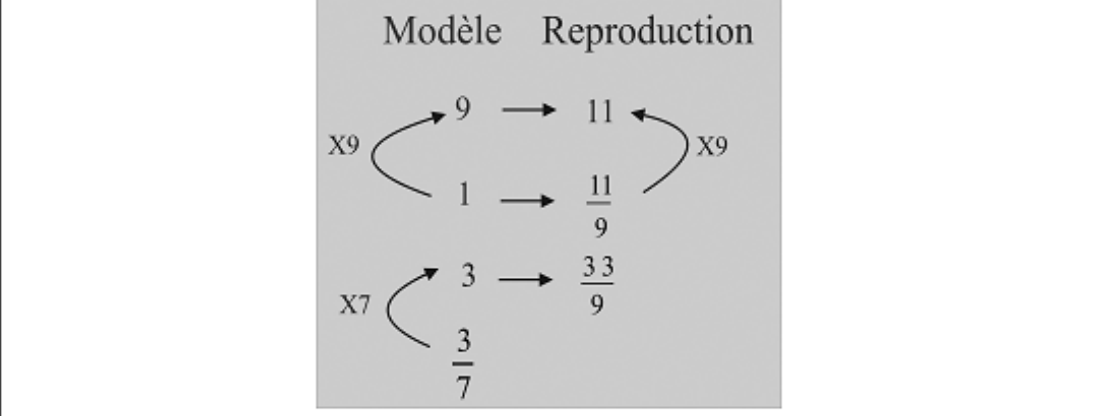 \\
\hline $\begin{array}{c}77.45- \\
80\end{array}$ & $618-621$ & $\begin{array}{l}\text { P rappelle en fin de séance ce qu'il faut savoir (institutionnalisation) à l'issue de } \\
\text { la séance. }\end{array}$ \\
\hline
\end{tabular}


Nous considérons par principe que toutes les phases qui constituent le découpage de la séance constituent des " jeux d'apprentissage ", au sens où le changement de phase correspond à une manière différente, pour le professeur, de jouer sur le jeu des élèves. Ceci nous conduit à ne pas préjuger de la nature des jeux considérés ni de l'implication des joueurs coopératifs que sont les élèves et le professeur. Nous allons donc les analyser un par un en cherchant à en déterminer la fonction génétique d'un milieu, d'une progression, d'une position selon le cas.

\section{Le jeu d'apprentissage générique « rappel "}

Ce jeu d'apprentissage ouvre la leçon. Il fait suite à une séance de résolution de problème où les élèves ont dû rechercher l'image de la mesure 7 pour la reproduction d'un puzzle dont la reproduction est quatre fois plus petite.

1. P: Est-ce que quelqu'un pourrait venir nous montrer? // Guillaume? // oui // alors écris-le // Alors vous vous souvenez hein // alors on a dit il faut montrer comment on a fait // alors tu mets quelque chose pour qu'on comprenne comment tu fais //oui // alors pour bien montrer d'où on part et où on va qu'est ce qu'on a intérêt à mettre

2. Elv/xx: des flèches

3. P: ouais tu as entendu ce qu'on vient de dire guillaume?

4. Elv /bl : ah oui des flèches

5. $\mathrm{P}$ : des flèches // des flèches de l'autre côté

6. Elv /bl : de l'autre côté aussi?

7. P: ben oui

8. Elv /bl : et là aussi

9. $\mathrm{P}$ : et là aussi

10. Elv/el: P P je vois pas ca brille

11. P: ah ça brille un peu ben // écoute tu te voilà tu te bouges un peu //oui alors nous avions trouvé

12. Elv/bl : un virgule soixante-quinze

Au tour de parole 1, le travail réintroduit un système sémiotique, qui va donner à voir et à comprendre l'usage d'un modèle mathématique notamment avec le « où on part et où on va », le « il faut montrer » ou le « bien » (tdp.1: « alors on a dit il faut montrer comment on a fait; alors pour bien montrer d'où on part et où on va qu'est ce qu'on a intérêt à mettre »). Ce système pourra donc figurer désormais dans répertoire des élèves pour tous les jeux à venir, il appartient donc au milieu des situations à venir: le geste du professeur est mésogénétique.

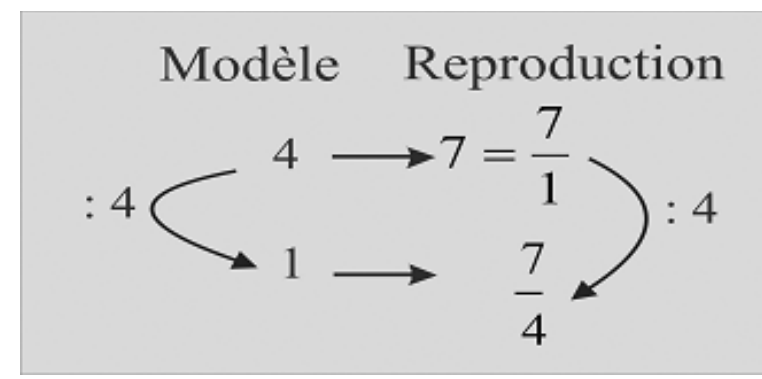

La mésogenèse s'appuie sur les normes pérennes $\mathrm{du}$ contrat didactique pour introduire l'objet nouveau: "Alors vous vous souvenez hein..." , où le régulateur d'assentiment " hein » apporte une certaine marque de complicité. L'usage des pronoms permet de décrire une topogenèse très complexe, où $\mathrm{P}$ est tantôt dans une position haute, lorsqu'il demande une action: (tdp.1: alors tu mets; tdp.8: et là aussi) tantôt dans une position basse lorsqu'il appelle à une réflexion: (tdp.1: ouais tu as entendu ce qu'on vient de dire guillaume?; tdp.11: oui alors nous avions trouvé). Ces mouvements topogénétiques ont pour effet de permettre à bl de jouer en position basse attendant les instructions de P: (tdp.1: Elv/b1: de l'autre côté aussi ?; tdp.1: Elv /b1 : de l'autre côté aussi? ).

13. P : c'est ça // est ce que tu pourrais expliquer comment vous aviez trouvé ça? // comment on peut trouver un virgule soixante-quinze comment on fait sept divisé par quatre

14. Elv/xx: parce qu'on avait plus

15. Elv /bl : en faisant en fraction

16. P: oui alors on met en fraction alors comment on met sept en fraction? oui attend tu vas écrire sur le côté ça sera mieux hein

17. Elv /el : j'y vois rien

18. $\mathrm{P}$ : sept pour un // appuie bien // tu vois pas?

19. Elv /el : à cause de des reflets

20. $\mathrm{P}$ : divisé par quatre // alors tu écris tout // sept pour un divisé par quatre égal alors écris le d'abord en fraction // alors sept sur un divisé par quatre égal // allez qui peut aller lui écrire?

21. Elv/bl : ben sept fois quatre

Les élèves ont finalement trouvé la mesure 1,75. En 13, P sollicite la restitution collective des actions accomplies pour trouver ce résultat: (est ce que tu pourrais expliquer comment vous aviez trouvé ça // comment on peut trouver un virgule soixante-quinze comment on fait sept divisé par quatre). 


\begin{tabular}{|l|}
\hline 22. P: oh si tu sais le donner directement // oui tu fais la \\
transformation // si tu veux allez vas-y comme ça on va bien \\
montrer tout ce qu'on peut faire // il y en a qui savent le donner \\
directement \\
\hline 23. Elv /xx: oui /bl sept sur quatre \\
\hline $\begin{array}{l}\text { 24. P: alors ça c'est la transformation oui égal sept pour quatre } \\
\text { appuie sur ta craie sinon on verra pas voilà et après on a essayé } \\
\text { d'écrire en nombre à virgule }\end{array}$ \\
\hline 25. Elv /bl: fois \\
\hline $\begin{array}{l}\text { 26. P: vas y écris en dessous alors comment on peut passer } \\
\text { voilà là sept pour quatre si tu l'écris en nombre à virgule }\end{array}$ \\
\hline 27. Elv /bl : ben sur cent \\
\hline $\begin{array}{l}\text { 28. P: allez qui peut l'aider allez Guillaume qui veut aller l'aider } \\
\text { je veux écrire en nombre à virgule pour montrer comment vous } \\
\text { avez fait hier? oui }\end{array}$ \\
\hline 29. Elv /xx: on a fait une transformation \\
\hline 30. P: on a fait une transformation alors par combien \\
\hline 31. Elv: /bl sur cent \\
\hline
\end{tabular}

En transformant $7 / 4$ en fraction décimale, (tdp.29: Elv/xx: on a fait une transformation) les élèves ont pu écrire le résultat des mesures obtenues pour la reproduction sous la forme décimale.

32. P: oui sur cent alors par combien on a transformé?

33. Elv/bl : vingt-cinq

34. P : oui alors marque-le // voilà en haut aussi // allez on marque tout // combien ça fait on compte dans sa tête // voilà on est d'accord? //oui ça va // on est tous d'accord // alors on va le voir vous sauriez le refaire pour euh par exemple euh un autre puzzle?

35. Elv/xx: oui /xx oui

36. P: oui et ben on va voir // tu distribues ici une feuille // tu distribues ici une feuille pour chacun tu distribue ici une feuille // allez mettez de suite votre nom comme ça // ça y est tu as mis ton nom Olivier?

37. Elv /e1: oui

Le tour de parole 34 avec le « on est tous d'accord » vient confirmer l'arrière-plan sur lequel les élèves vont devoir procéder pour la recherche d'autres transformées de mesures (oui alors marque-le // voilà en haut aussi // allez on marque tout // combien ça fait on compte dans sa tête // voilà on est d'accord? //oui ça va // on est tous d'accord // alors on va le voir), c'est-à-dire des mesures exprimées sous forme décimale. Ainsi l'introduction d'un objet dans le milieu (le système sémiotique instruisant des stratégies des transformées de mesures) transformation, qui signe un jeu mésogénétique, fait avancer le temps en montrant le nouveau de la situation du jour et a donc une fonction chronogénétique à l'ouverture de la séance. C'est dans ce milieu que le contrat didactique va se construire conjointement par le professeur et les élèves.

\section{Le jeu d'apprentissage « Image d'un nombre entier"}

À la suite d'une intervention en aparté entre un chercheur présent et le professeur qui conduit la séance, ce dernier vient de proposer la recherche de l'image d'une nouvelle mesure entière aux élèves. Le travail mésogénétique de $\mathrm{P}$ va maintenant consister à ré-orienter l'action conjointe vers la commensuration ${ }^{4}$ :

76. P: comment non si je vois que tout le monde a une réponse // réfléchis bien ça y est allez on y va? allez on pause son crayon // Sophie tu vas au tableau? // tu peux prendre ta feuille on t'écoute dis-nous comment tu as fait

77. Elv/c2: j’ai fait neuf divisé par neuf pour le modèle

78. P: c'est-à-dire c'est parce que c'est comment un par rapport à neuf?

79. Elv/xx: neuf neuf fois plus petit

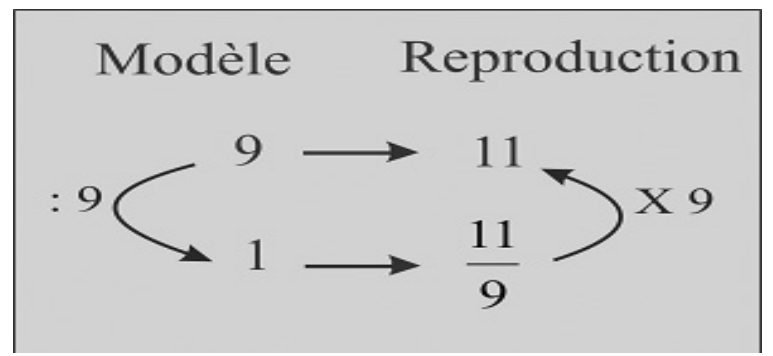

En 77 l'élève c2 indique l'arrière-plan de la transaction didactique (tdp.77: j'ai fait neuf divisé par neuf pour le modèle) que P lui demande de préciser par le « c'est-à-dire » et le "parce que » du tdp.78 (c'est-à-dire c'est parce que c'est comment un par rapport à neuf?).

80. P : c'est neuf fois plus petit vas-y un c'est neuf fois plus petit que neuf sur le modèle alors sur la reproduction le nombre qu'on va trouver ici sur la reproduction

81. Elv /cl : il va être neuf fois plus petit

82. P: il va être

83. Elv/xx: neuf fois plus petit

84. P : neuf fois plus petit que onze vous êtes d'accord? oui donc si je prends dans l'autre sens onze c'est comment c'est comment par rapport à ça?

85. Elv/xx: neuf fois plus grand neuf fois plus grand (collectif)

86. $\mathrm{P}$ : neuf fois plus grand alors qu'est ce qui quand j'en prends neuf est égal à onze comment ça s'écrit ça?

87. Elv/cl: en fraction

$\mathrm{P}$ utilise alors les ressources offertes par le système sémiotique pour ré-orienter l'action possible dans le milieu. Avec le « alors qu'est-ce qui » et grâce à l'énoncé de reconnaissance des fractions connue 
des élèves de: "alors qu'est ce qui quand j'en prends neuf est égal à onze comment ça s'écrit ça? » (tdp.86), il introduit ainsi la commensuration dans l'arrièrefond de la transaction didactique. Dans ce travail mésogénétique de retour à la référence de la situation fondamentale (qui signifie par exemple que si j'en prends 9 ça fait 11), l'usage des pronoms ((84) si je prends dans l'autre sens; (86) quand j'en prends neuf), place $\mathrm{P}$ est en position haute.

\begin{tabular}{|l|}
\hline $\begin{array}{l}\text { 88. P: oui écris-le en fraction ça s'écrit est ce que vous êtes } \\
\text { d'accord? }\end{array}$ \\
\hline 89. Elv /xx: oui \\
\hline $\begin{array}{l}\text { 90. P: alors j'ai vu qu'il y en avait qui étaient un peu coincés } \\
\text { là par ce qui // oui // qu'est ce que tu mettrais ici? // est ce que } \\
\text { vous êtes d'accord avec Sophie c'est ça? est ce que ça corres- } \\
\text { pond bien avec ce qu'on connaît sur la fraction? }\end{array}$ \\
\hline 91. Elv /xx: oui \\
\hline $\begin{array}{l}\text { 92. P: est ce que cette façon-là c'est bien l'écriture de quelque } \\
\text { chose telle que telle que }\end{array}$ \\
\hline 93. Elv/cl: si j'en prend \\
\hline 94. P: oui si j'en prends \\
\hline 95. Elv/cl: si j'en prends neuf \\
\hline 96. P: ça \\
\hline 97. Elv/cl: ca fait onze? \\
\hline
\end{tabular}

En 90, l'usage du « on » marque un mouvement topogénétique descendant ${ }^{5}$ (est-ce que ça correspond bien avec ce qu'on connait sur la fraction?). Le travail mésogénétique consiste alors à faire jouer aux élèves la transaction didactique dans le «langage des fractions-mesure ». En 92, P revient à l'énoncé caractéristique des situations de mesure avec les fractions (tdp.92: est-ce que cette façon-là c'est bien l'écriture de quelque chose telle que telle que) et laisse la poursuite de l'énoncé dans le topos de cl par réticence ${ }^{6}$ didactique (Sensevy \& Quilio, 2002) (tdp93. : si j'en prends). Ce phénomène de réticence didactique se poursuit, il engage et autorise le retour au jeu avec les premières écritures de fractions (P92: est ce que cette façon-là c'est bien l'écriture de quelque chose telle que telle que $\rightarrow$ Elv/cl : si j'en prends $\rightarrow$ P94: oui si j'en prends $\rightarrow$ Elv /c1: si j'en prends neuf $\rightarrow$ P96: ça $\rightarrow$ Elv/cl : ça fait onze?)

98. P: on est d'accord? ça correspond bien à ce qu'on connaît
sur la fraction?
99. Elv /cl : oui
100. P: oui alors il y en a qui étaient coincés qui me disent je
n'y arrive pas parce que je sais pas donner l'écriture // comment
en est ce que c'est important,
101. Elv/xx: non
102. P: est ce que ça c'est bien aussi une mesure?

103. Elv/xx: oui oui (collectif)

104. P: oui ben c'est une réponse // vous la donnez sous forme de fraction // on pourrait si on voulait la donner sous forme de nombre décimal // mais on peut la donner sous forme de fraction c'est ça qui vous gênait?

105. Elv/xx: oui oui (collectif)

Au tour de parole 100 et 104 l'usage des pronoms montre $\mathrm{P}$ dans un mouvement topogénétique ascendant $^{7}$ (Tdp.100: alors il y en a qui étaient coincés qui me disent je n'y arrive pas; tdp1004. : oui ben c'est une réponse // vous la donnez sous forme de fraction), et le travail de mésogenèse s'achève par la formulation des actions possibles dans le milieu (tdp.104: mais on peut la donner sous forme de fraction c'est ça qui vous gênait?). Le professeur réintroduit dans le milieu l'épaisseur sémantique qui provient des rapports aux fractions construits depuis le commencement de l'enseignement, ce qui est cette fois un travail mésogénétique sans effet chronogénétique. Le contrat didactique va pouvoir reposer sur l'établissement de significations communes permettant la construction de stratégies d'actions possibles.

\section{Le jeu d'apprentissage « Recherche de la référence "}

Les élèves viennent de chercher l'image d'une mesure entière. Ils vont devoir par la suite chercher l'image d'une mesure fractionnaire.

\begin{tabular}{|l|}
\hline 218. P: mais un entier alors est ce que les mesures c'est toujours \\
forcément des nombres entiers? \\
\hline 219. Elv/xx: non \\
\hline 220. P: on avait vu d'autres \\
\hline $\begin{array}{l}\text { 221. Elv/c4: lorsqu'on avait vu deux c'était trois virgule cinq } \\
\text { c'était pas un nombre entier }\end{array}$ \\
\hline $\begin{array}{l}\text { 222. P: bon il y a des nombres comme ça et qu'est ce qu'il y a } \\
\text { d'autre aussi comme mesure? les mesures qu'est ce que ça peut } \\
\text { être? ça peut être des nombres entiers ça peut être quoi? }\end{array}$ \\
\hline 223. Elv /xx: des virgules \\
\hline 224. P: des nombres à virgule et ça peut être quoi encore? \\
\hline 225. Elv/xx: des fractions \\
\hline $\begin{array}{l}\text { 226. P: des fractions est ce que vous vous souvenez à quelle } \\
\text { occasion on a on a eu des mesures avec des fractions? }\end{array}$ \\
\hline 227. Elv /f1: euh là ceux-là qu'il disait là \\
\hline 228. P: oui \\
\hline $\begin{array}{l}\text { 229. Elv/f1: c'est ceux-là comme il disait là faut multiplier les trucs } \\
\text { comment faire/xx quoi j'entends pas /fl là je me rappelle pas }\end{array}$ \\
\hline $\begin{array}{l}\text { 230. P: alors qui j'ai entendu ici quand est ce qu'on a utilisé } \\
\text { les fractions }\end{array}$ \\
\hline 231. Elv/c4: les feuilles les paquets de feuille \\
\hline $\begin{array}{l}\text { 232. P: les paquets de feuilles et puis aussi on a mesuré des } \\
\text { petites baguettes est ce que vous vous souvenez }\end{array}$ \\
\hline
\end{tabular}


Le travail va préciser les ressources du milieu pour les actions à venir, c'est-à-dire la fractionmesure. Ce travail ouvre la chronogenèse de la séance et repose sur la mémoire du système didactique que $\mathrm{P}$ partage avec les élèves (P 220: on avait vu d'autres). Dans ce travail, $\mathrm{P}$ sélectionne conjointement avec les élèves (dans la mémoire du système didactique) la notion qu'il veut voir apparaître dans le milieu (tdp.222: P : bon il y a des nombres comme ça et qu'est ce qu'il y a d'autre aussi comme mesure? les mesures qu'est ce que ça peut être? ça peut être des nombres entiers ça peut être quoi?; tdp.224. P: des nombres à virgule et ça peut être quoi encore?) et qu'il introduit en s'appuyant sur un élève, mais aussi et surtout la situation fondatrice des pratiques associées, la mesure de l'épaisseur des feuilles mises en paquets qu'il relie à la question des «petites baguettes» que l'on mesure aussi.

\section{Elv/xx: ah oui ah oui}

234. P: je vous en avais donné plein on avait mesuré des longueurs de baguettes

235. Elv/c4: ah oui sur la feuille il y en avait plein

236. P: voilà vous vous souvenez par exemple si je vous dis celle-ci là trois trois trois huitièmes trois pour huit la mesure trois pour huit si qu'est ce que ça serait si c'était une baguette

237. Elv /bl : c'est trois

238. P : alors oui elle est telle que si j'en prends

\section{Elv /e 4 : huit ca fait trois}

240. P : ça fait trois unités ou bien si c'était les feuilles la feuille elle est épaisse telle que

241. Elv /e4: si j'en prends huit ça fait trois millimètres (en cour avec la classe)

P prend soin de jouer avec les élèves le jeu d'apprentissage qu'il vient de mettre en place dans le « langage des fractions » (tdp.238: P: alors oui elle est telle que si j'en prends; tdp.239: Elv le4: huit ça fait trois), un jeu sur la commensuration. Il précise ainsi le système stratégique disponible lié au contrat didactique.

\section{Le jeu d'apprentissage "Image d'une mesure exprimée en fraction "}

Le professeur fait le tour de toutes les recherches en cours auprès des élèves. Il remarque beaucoup de confusions concernant l'usage du système sémiotique. Il prend alors la parole devant le collectif.
267. P: ne mélanger pas les mesures sur le modèle et les mesures sur la reproduction ça avance? tu as discuté avec Jérémie alors tu le fais allez on va faire une pause et on va voir où on en est allez on pose les stylos ça y est on fait le point chut Nadia tu y es Morgan tu y es Caroline allez on en est là je vous ai donné comme renseignement ici c'est les mesures sur le modèle ici les images sur la reproduction les mesures sur la reproduction ça va on vous a dit je vous ai donné comme renseignement neuf sur le modèle a pour image onze sur la reproduction vous avez dit je cherche pour un parce que si je sais pour un je vais pouvoir me débrouiller et vous avez trouvé un sur le modèle a pour image onze pour neuf sur la reproduction ça va je vous demande maintenant quelle est si j'ai comme mesure trois pour sept là sur le modèle quelle est son image sur la reproduction c'est clair oui ? oui alors j'ai vu quelques idées Guillaume euh Farid s'il te plaît tu poses ton stylo et tu es tout les yeux et les oreilles là vers ici

Avec le « on va faire une pause », $\mathrm{P}$ annonce un recentrage qui va porter sur les actions possibles dans le milieu. Ce travail focalise l'attention sur les règles d'action que les élèves doivent mettre en œuvre (vous avez dit je cherche pour un parce que si je sais pour un je vais pouvoir me débrouiller et vous avez trouvé un sur le modèle a pour image onze pour neuf sur la reproduction ça va). Il précise ce qui est du topos de chacun:

Pour le professeur: "je vous ai donné comme renseignement ici c'est les mesures sur le modèle ici les images sur la reproduction les mesures sur la reproduction; je vous demande maintenant quelle est si j'ai comme mesure trois pour sept là sur le modèle quelle est son image sur la reproduction c'est clair oui ».

Pour les élèves: "vous avez dit je cherche pour un parce que si je sais pour un je vais pouvoir me débrouiller».

Dans ce travail qui ralentit le temps didactique afin de garantir la capacité d'action des élèves dans le milieu et qui est donc mésogénétique, un énoncé tel que "vous avez dit » constitue en quelque sorte un antidote au dialogue de sourds dans la transaction didactique en cours. Le jeu d'apprentissage a donc ici été arrêté afin d'être re-contractualisé plus précisément. Ce genre d'intervention est en général le signe d'un arrêt de la progression dû à une difficulté trop grande, qui fait obstacle et qu'il va falloir franchir ou contourner. Elle montre que dans le jeu didactique, le milieu, comme potentiel d'actions pour les élèves, nécessite pour le professeur et les élèves de partager des stratégies disponibles qui sont constitutives du contrat didactique. 
Ce phénomène montre aussi la grande difficulté de mise en œuvre de ce type de situations d'enseignement par la grande vigilance épistémologique qu'elles exigent de l'enseignant qui les met en œuvre.

\section{Le jeu d'apprentissage «Image d'une mesure exprimée en fraction " épisode "l'intermédiaire $1 / 7$ "}

L'analyse précédente nous conduit à reconsidérer le jeu de l'intermédiaire $1 / 7$ soit, comme un épisode du jeu «Image d'une mesure exprimée en fraction, 3/7 » qui est de fait, arrêté, soit comme un jeu de substitution proposé par le professeur en remplacement du précédent qui faisait obstacle. La suite nous dira qu'elle est, des deux, l'interprétation pertinente. Car les élèves ont fait une première tentative individuelle de recherche de l'image de la mesure $3 / 7$, mais certains rencontrent beaucoup de difficultés, et même, $\mathrm{P}$ a écrit $7 / 7$ sur le tableau de mesures pour leur permettre de trouver plus facilement l'opérateur à appliquer pour trouver $1 / 7$.

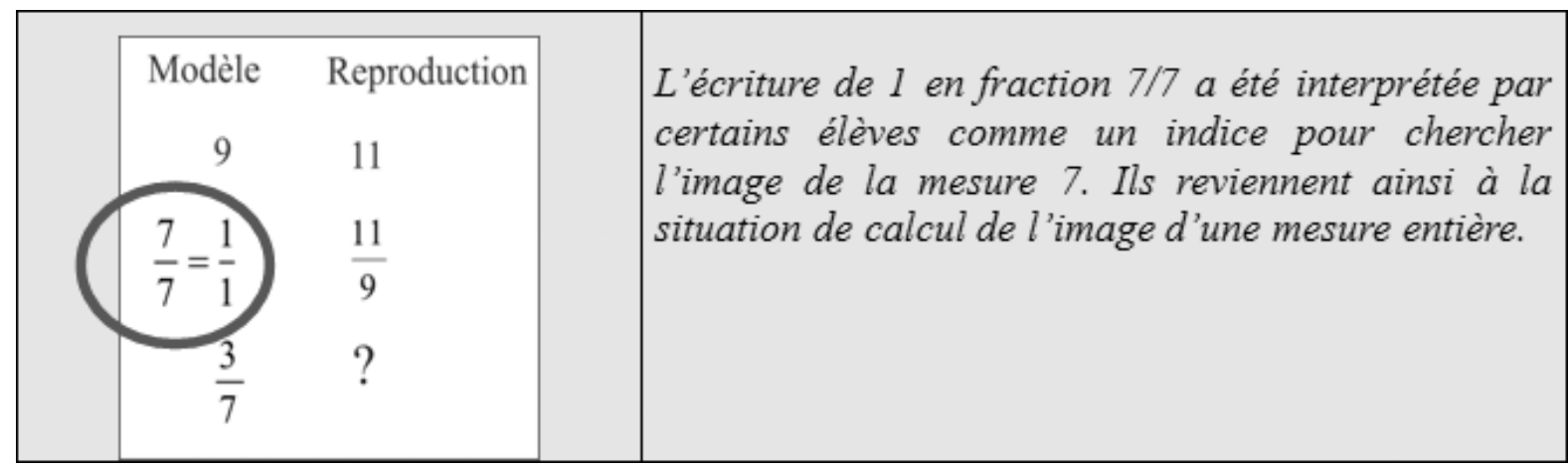

313. P: moi j'ai vu des idées là dans les dans les cahiers alors si si on reprend ici personne n'a d'idée comment on peut passer on sait pas passer de sept septièmes à trois septièmes // est ce qu'on pourrait pas trouver un intermédiaire qui nous permettrait après de repasser à trois septièmes

314. CR: mais avec trois aussi on pourrait alors essayez allez

315. Elv/c4: nous on a fait

316. P: vous vous avez eu une idée alors qu'est ce que vous avez cherché vous?

317. Elv/c4: on a fait pour sept et après

318. P: vous avez cherché pour sept d'abord

319. Elv/c4: oui

$\mathrm{P}$, en 313 , avec le « après » précise le rôle de l'intermédiaire qui est de permettre de passer facilement à trois septièmes (tdp.313: est-ce qu'on pourrait pas trouver un intermédiaire qui nous permettrait après de repasser à trois septièmes). Cet intermédiaire est facilement identifiable si on écrit la mesure 1 en une mesure équivalente 7/7. Mais l'effet Topaze complet engage le professeur à expliquer... son indication!

320. P : pourquoi vous avez vous avez eu l'idée de chercher pour sept?

321. Elv/c4: parce que il y avait le le sept du dénominateur

322. P : et qu'est ce qu'il signifie le sept du dénominateur? le sept d'ici?
323. Elv /c4: sept sept septièmes

324. $\mathrm{P}$ : il signifie quoi ? que c'est des

325. Elv: septièmes

326. P: c'est des septièmes alors qu'est ce que c'est que des septièmes

327. Elv/c4 : c'est des

328. P: c'est quelque chose // qu'est ce que c'est que des septièmes? vous dites c'est des septièmes c'est quoi des septièmes?

En $320,322,324$, P occupe une position topogénétique haute sans réussir à obtenir des élèves qu'ils sortent de "l'ahurissement " provoqué par son indication (tdp.320: P : pourquoi vous avez vous avez eu l'idée de chercher pour sept?; tdp.322: et qu'est ce qu'il signifie le sept du dénominateur? le sept d'ici?; tdp.324: il signifie quoi? que c'est des). Le tour de parole 328 est caractéristique. En effet en 328 , le "c'est quelque chose // qu'est ce que c'est que des septièmes? » est une première tentative de $\mathrm{P}$ pour faire émerger un élève au moins.

\begin{tabular}{|l|}
\hline 329. Elv /d4 : ben c'est \\
\hline 330. Elv /c4 : c'est \\
\hline 331. P: c'est? \\
\hline 332. Elv /c4: c'est sept fois plus petit que sept unités \\
\hline
\end{tabular}


333. P: que quoi c'est sept fois plus petit que quoi le septième c'est sept fois plus petit que quoi le septième 334. Elv/c4: que sept unités

335. P: que sept unités? sept fois plus petit que sept unités? chez moi c'est combien? quelque chose qui est sept fois plus petit que sept unités ben enfin

336. Elv/c4: un

Mais le sens de l'indication n'est pas compris (tdp.333: P : que quoi c'est sept fois plus petit que quoi le septième // c'est sept fois plus petit que quoi le septième) par c4 (tdp.329: Elvc/4: ben c'est). P tente maintenant de faire dire que $7 / 7=1$, mais c'est déjà écrit et les élèves ne se décident pas à jouer ce jeu.

Le «chez moi» du tour de parole 335 marque un rappel explicite d'un contrat plus général sur les entiers et leurs multiples qui permet à c4 de répondre, ce que le professeur prend pour l'accès de l'élève au sens de septième et de 7/7 (tdp.336).

337. P: oui c'est un // alors c'est sept fois plus petit qu'une unité // quelque chose telle que si j'en prends

338. Elv /el : sept

339. P: sept // ca fait une unité // comment ca s'appelle? oui ça s'appelle

340. Elv/d2: un septième

341. P: oui un septième et comment ça s'écrit ça?

342. Elv/c4: un pour sept

343. P: oui viens l'écrire viens l'écrire ici ah chut chut ah Guillaume il sait déjà écris moi là qu'est ce que ça signifie ça?

344. Elv /c4: un septième

345. P: un septième c'est quelque chose

346. Elv: /b2 si j'en prends

347. P: oui telle que si j'en prends sept ça fait une unité alors est ce qu'il lui est euh c'est ca qui qui l'a interpellée elle dit elle s'est dit je vais faire quelque chose avec ce sept là

Ce travail permet enfin de mobiliser le jeu de langage fondamental des fractions. Le travail mésogénétique a donc abouti et il permet de disposer enfin dans la classe d'un arrière-plan commun P: oui viens l'écrire viens l'écrire ici ah chut chut ah Guillaume il sait déjà écris moi là qu'est ce que ça signifie ça?). La réticence didactique peut alors fonctionner (tdp.345 $\rightarrow$ tdp.346.) et le professeur arrive à orienter l'action publique, au tableau, des élèves qui se manifestent et qu'il appelle à jouer le jeu.

\begin{tabular}{|l|}
\hline 348. Elv/bl: fois trois /el fois trois /d4 on fait fois trois \\
\hline 349. P: alors quoi fois trois? \\
\hline 350. Elv/d4: un septième \\
\hline 35l. P: pourquoi \\
\hline 352. Elv/d4: on fait un un pour sept fois trois \\
\hline
\end{tabular}

353. P : alors trois pour sept c'est comment par rapport à un pour sept?

354. Elv /f1 : euh trois euh c'est trois fois plus grand

355. P: c'est trois fois plus grand c'est un pour sept un pour sept un pour sept bon alors est ce que vous sauriez trouver alors

356. Elv/el: oui fois trois

357. P : pour un pour d'accord mais j'ai pas fini vous me dites si je sais trouver pour un pour sept je saurais trouver après pour trois pour sept

358. Elv/xx: oui

Cet arrière-plan commun permet en effet aux élèves d'opérer les actions convenables sur les objets du milieu (tdp.352: Elv/d4: on fait un un pour sept fois trois): le jeu peut être joué. Les actions disponibles dans le milieu sont maintenant partagées par le professeur et ses élèves et inscrites dans le contrat didactique.

\section{Le jeu d'apprentissage "Image d'une mesure exprimée en fraction " devenu «Transformation d'une fraction-mesure "}

La résolution de la question pour $1 / 7$ change l'enjeu du jeu initial. P organise d'abord la mise en commun après la recherche individuelle de l'image de 1/7 et c'est en ce point que l'on observe élèves et professeur face à l'enjeu de la leçon. Pour une meilleure compréhension des échanges qui suivent, je compléterai les échanges du système sémiotique utilisé par le professeur sur le tableau de la classe.

432. P: je ne veux pas que tu me mettes des calculs ici en même temps // tu mets la flèche //attention// top posez les stylos //c'est fini c'est fini //je vois plus personne écrire// terminé// Estelle tu viens ici// attention// Olivier //on a dit un septième c'est sept fois plus petit que sept septièmes // sept septièmes c'est un // si c'est sept fois plus petit sur le modèle//ça doit être comment sur la reproduction?

\section{Elv/d1 : sept fois plus petit}

434. P : sept fois plus petit sur la reproduction//alors quelque chose sept fois plus petit que onze neuvièmes// comment on peut l'écrire //ici// sept fois plus petit comment ça peut s'écrire? 435. Elv /b2: divisé par sept

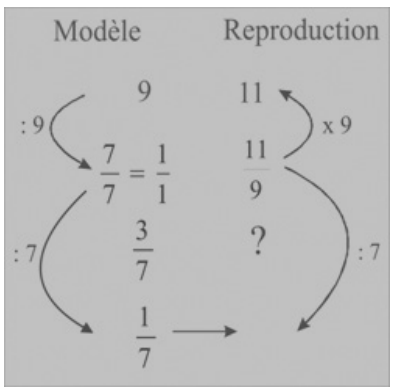


L'élève b2 est appelée au tableau pour participer à la correction collective (tdp.432) et écrire les résultats dans le tableau de mesures.

436. P: oui divisé par sept //allez tu l'écris// là //c'est le calcul
qui l'embête Estelle //c'est pas grave// c'est ça que tu sais plus
faire // ben c'est pas grave //on va t'aider //alors le calcul c'est
quoi// on va se faire un un cahier de brouillon ici au milieu//
regardez c'est ça qui vous embête qui vous coince //c'est rien du
tout //c'est le calcul //alors on écrit ici pour trouver la réponse
d'accord c'est un cahier de brouillon //de calcul//alors comment
on fait// tu te souviens plus comment on fait pour div //et ben
c'est pas grave //qui est ce qui peut l'aider ?// lui rappeler// chut
//tu te souviens des feuilles des feuilles //il en il en faut //chut
chut //va pas y passer beaucoup de temps quand c'était un tas
de feuilles on disait
437. Elv/d3: (inaudible)

Le tour de Parole 436 est exemplaire du travail de régulation du jeu, qui distingue l'erreur de calcul de l'erreur conceptuelle. Le professeur peut maintenant s'engager sur ce chemin, parce qu'il l'a viabilisé comme on le voit avec l'appel aux feuilles de papier qui est donc la condition sine qua non. Le « divisé par 7 » suggère un retour possible des élèves à la conception partage contre la conception commensuration. La succession de "c'est pas grave; c'est rien du tout; qui peut l'aider » indique clairement aux élèves une attente faible du professeur pour la procédure de calcul.

438. P : bon et ici quand on divisait par sept ça voulait dire que le nombre là il est tel que quand je le multiplie par sept je dois retrouver

439. Elv/d3: neuf pour onze

440. P: onze pour neuf ça va? tu te souviens du sens? bon alors je crois qu'elle ne se souvient plus de la de la technique du calcul

441. Elv /xx: je peux arriver à la faire moi ça

442. P: qu'est ce qui multiplié par sept c'est ça qu'on cherche hein qu'est ce qui multiplié par sept est égal à onze pour neuf d'accord alors qu'est ce qu'on fait?

443. Elv /b4: une transformation

444. P: pourquoi une transformation pourquoi est ce que ce serait commode

445. Elv/b4: parce que neuf c'est pas dans la table de sept

446. P: non parce que onze

447. Elv: /bl onze c'est pas dans la table de sept

448. $\mathrm{P}$ : alors est ce que au lieu d'écrire onze neuvièmes tu pourrais écrire quelque chose telle que fois sept on trouve ici ça soit dans la table de sept on transforme alors comment ça s'écrit allez vas y voilà pour euh

449. Elv/d3: neuf (/d3 écrit soixante dix-sept sur neuf)

450. P: psitt psitt c'est une transformation

En 438 P revient sur le sens du mot « divisait » pour s'assurer de la relation idoine au milieu dans une manifestation de la réticence didactique ( $\mathrm{P}$ : ça voulait dire que le nombre là il est tel que quand je le multiplie par sept je dois retrouver $\rightarrow \mathrm{d} 3$ : onze pour neuf). Le «neuf pour onze » du tdp.439 satisfait les attentes de $\mathrm{P}$ qui portent maintenant sur la procédure de calcul qui permet d'obtenir ce qui multiplié par 7 donne 11/9 (tdp.444: pourquoi une transformation ce serait commode).

\begin{tabular}{|l|}
\hline 451. Elv: /xx soixante-trois \\
\hline $\begin{array}{l}\text { 452. P: ah oui la transformation attention voilà bon alors qu'est } \\
\text { ce qui maintenant on y est? vous avez été plusieurs à qu'est } \\
\text { ce qui multiplié par sept est égal à soixante-dix-sept pour } \\
\text { soixante-trois? }\end{array}$ \\
\hline 453. Elv /xx: pour neuf \\
\hline 454. P: ah attention multiplié qu'est ce qui multiplié par sept? \\
\hline 455. Elv /d3: onze pour neuf \\
\hline $\begin{array}{l}\text { 456. P: tu dis onze pour neuf on essaie on vérifie d'accord elle } \\
\text { dit c'est onze pour neuf est ce que onze pour neuf fois sept ça } \\
\text { fait bien ça? }\end{array}$ \\
\hline 457. Elv: /xx non \\
\hline $\begin{array}{l}\text { 458. P: alors // prends ton temps // prends ton temps ça fois ça } \\
\text { et il faut qu'on trouve ça }\end{array}$ \\
\hline 459. Elv /d3: onze pour soixante-trois \\
\hline 460. P: voilà tu vois donc la réponse ici c'est \\
\hline 461. Elv/d3: onze pour soixante-trois \\
\hline 462. P: allez écris le alors l'image de un septième c'est? \\
\hline 463. Elv/d3: onze pour soixante-trois \\
\hline $\begin{array}{l}\text { 464. P: voilà est ce que c'est bien très bien est ce que si je fais } \\
\text { dans l'autre sens est ce que onze pour soixante-trois fois sept est } \\
\text { ce que je retrouve bien onze pour neuf? }\end{array}$ \\
\hline
\end{tabular}

On le comprend, l'écriture d'une équation ou d'un modèle algébrique de la situation manque ici et c'est ce qui oblige professeurs et élèves à des jeux de langage complexes. Dans ce travail, P marque une grande attention au défilement du temps. Il ralentit (tdp.458: prends ton temps), rassure (tdp.460: voilà tu vois; tdp.464: c'est bien c'est très bien) mais garde toujours une vigilance conceptuelle sur le milieu de la situation (tdp.464: est ce que si je fais dans l'autre sens est ce que onze pour soixante-trois fois sept est ce que je retrouve bien onze pour neuf?). Car le travail réalisé ici nourrira longtemps encore la progression collective et il faut donc que tous les élèves aient remarqué qu'en ce point ils franchissent un stade et entrent dans un monde nouveau. Cette nouveauté doit être reconnue et acceptée.

\section{Elv: /xx oui}

466. P: oui vous êtes sûrs? bon alors dites donc on a maintenant l'image maintenant de un septième alors vous savez tous trouver l'image de trois septièmes? oui?

467. Elv: oui

468. P: trente secondes pour l'écrire sur votre cahier toi tu l'écris là tu te caches vous regardez pas et on vérifie 
Une fois la procédure de calcul évoquée pour chacun, le « trente secondes pour l'écrire » (tdp.438) marque une reprise de la progression, qui est maintenant dévolue aux élèves. Il marque l'établissement d'un contrat partagé pour traiter le problème.

\section{Le jeu d'apprentissage « Intermédiaire 3 "}

Ce jeu d'apprentissage se déroule à la fin de la séance. Il reste à faire apparaître la deuxième procédure qui permet d'obtenir 3/7.

514. P : alors je voudrais // qu'on juste avant de partir // Morgan il a fait autrement lui tout à l'heure //il a eu une autre idée je voudrais bien qu'on en parle// Morgan lui il s'est pas servi de un septième

515. Elv: /xx hein?/xx ah bon

516. P: il s'est servi d'autre chose

517. Elv/xx: il s'est servi de trois pour sept?

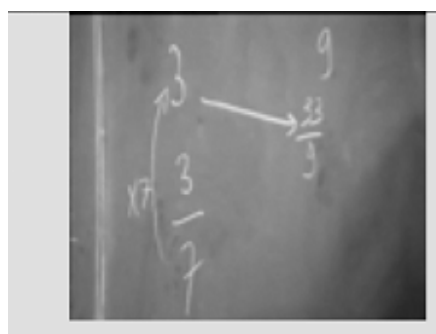

La description du jeu de Morgan dans le milieu s'effectue à partir d'un système sémiotique qui reproduit les différents coups du jeu consignés dans le cahier de Morgan (tdp.518: recopie ce que tu as écrit ici; tdp.520: on va l'écrire dans le même sens que sur ton cahier). $\mathrm{P}$ se substitue alors à Morgan et suspend la description du déroulement du jeu (tdp.520: attends dis rien // regardez ce qu'il a écrit est-ce que vous comprenez?).

522. P: pas trop // est ce que vous comprenez ce qu'il a écrit ici ? 523. Elv/xx: non /xx oui

524. P : la dernière chose qu'il a écrit qu'est ce qui est dit qu'est ce qui est dit là Maïlys

525. Elv/f1 : trois fois sept c'est égal à trois pour sept /c4 non 526. $\mathrm{P}$ : il dit que // t'écoutes // il dit que trois c'est sept fois plus grand que trois septièmes // ou bien il dit que trois septièmes fois sept c'est égal à trois est ce que c'est vrai

527. Elv/xx: hein

528. P : il dit trois septièmes fois fois sept c'est égal à sept 529. Elv/xx: oui c'est vrai

530. P : euh c'est égal à trois il dit moi je sais ça est ce que c'est vrai?
Dans ce jeu d'apprentissage, $\mathrm{P}$ va reconstituer la transaction didactique à partir de la démarche de Morgan. $\mathrm{P}$ prend une position topogénétiquement haute (je voudrai bien qu'on en parle). Le travail mésogénétique désigne un objet du milieu pour la transaction (tdp.514: Morgan lui il s'est pas servi de un septième).

518. P : allez //Morgan // au lieu de passer par un septième Morgan il est passé par? // écris le ici // recopie ce que tu as écrit ici // là ici // là oui

519. Elv/f3: j’ai fait trois comme ça

520. P : hum hum vas-y continue //après //alors moi j’ai écrit au-dessus mais ça fait rien //alors t'as écrit de trois septièmes à trois comme ça //non dans l'autre sens //tu l'as écrit tu veux que je te l'écrive pareil que sur ton cahier //alors // on va l'écrire dans le même sens que sur ton cahier //d'accord? // toi tu as écrit trois ici // allez vas-y // vas-y // et ici qu'est ce que tu as mis là // attends dis rien// regarde // regardez ce qu'il a écrit est ce que vous comprenez?

521. Elv /xx: non /xx non

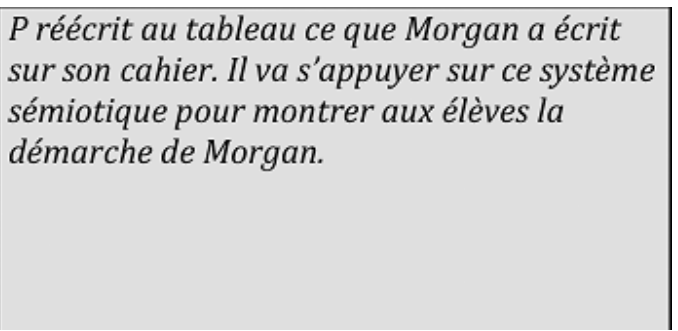

Le discours prend une forme narrative qui centre l'attention sur la stratégie de jeu suivie par Morgan (tdp.522: est-ce que vous comprenez ce qu'il a écrit; tdp.526: il dit que trois septièmes fois sept c'est égal à trois est-ce que c'est vrai; tdp.530: il dit moi je sais ça est-ce que c'est vrai). Cette forme permet au professeur d'intégrer cette stratégie dans le champ de la référence commune et donc d'instituer ce champ comme le moyen de penser et traiter, quoi qu'il en soit, les problèmes de ce type.

\section{Elv/xx: euh sept fois trois}

532. P : vous voulez vérifier? vous n'êtes pas d'accord sur ça? et ben alors faites-le //vérifiez //mettez vous ça dans dans un coin de votre cahier //est ce que c'est vrai ou c'est pas vrai ? // vérifiez avant de parler vérifiez s'il vous plaît

533. Elv /xx: ah

534. P : et alors alors combien vous trouvez vous? //alors s'il te plaît Natacha tu trouves combien toi?

535. Elv/el : vingt et un pour sept

536. P: j'ai pas entendu

537. Elv/e3: vingt et un pour sept 
538. $\mathrm{P}: \mathrm{ah}$

539. Elv:/fl oui mais d'accord mais comme c'est dans la table de sept 540. P : est-ce que vingt et un pour sept

541. Elv: /xx oui /xx oui

542. P : c'est trois

543. Elv/f1: oui /xx oui /xx oui c'est juste

$P$ poursuit en faisant vérifier la règle instituée par les élèves de la classe (tdp.532: et ben alors faites-le/l vérifiez// vérifiez avant de parler s'il vous plaît) afin de faire valider les actions permises dans le milieu et leur validité.

544. P : c'est juste? // mais dites donc on le savait déjà rien qu'en le voyant écrit //rien qu'en le voyant écrit// trois septièmes trois pour sept qu'est-ce que c'est que trois pour sept

545. Elv /al : euh euh

546. P : c'est quelque chose oui

547. Elv/d2: telle telle que si j'en prends sept

548. P: alors dit le bien chut c'est quelque chose telle que si

549. Elv /d2: j'en prends sept // j'en prends sept euh sept euh sept euh

550. P: oui vas-y

551. Elv /d2: sept j'en prends ça fait trois unités

552. P: Est-ce que vous avez entendu?

553. Elv/xx: non

554. P: je suis sûre que Caroline n'a pas entendu allez encore plus fort // j'ai rien entendu // fort

555. Elv/d2: si on prends sept

556. $\mathrm{P}$ :oui

557. Elv /d2: ça fait trois unités

558. $\mathrm{P}$ : je répète?

560. Elv/xx: oui

561. P : elle dit c'est quelque chose telle que si j'en prends sept

562. Elv/fl : sept

563. P : ça fait trois unités et ben est ce que c'est vrai?

564. Elv/xx: oui

565. P : c'est quelque chose telle que si j'en prends sept ça fait trois unités il a raison Morgan il l'avait vu rien qu'à l'écriture de la fraction

566. Elv/xx: il l'avait vu Morgan

567. P : et oui vous aviez oublié que la fraction ça a le sens que si j'en prends sept ça fait trois unités alors il a raison oui donc

il dit si je chut chut chut ça y est? alors comment il a trouvé

l'image de trois oh

Le fin du travail de P s'achève par un effet de contrat qui est un effet d'institutionnalisation (tdp.544: on le savait déjà rien qu'en le voyant écrit... qu'est-ce que c'est que trois pour sept). C'est donc sous l'égide du contrat didactique que s'exerce dorénavant l'énonciation caractéristique de la commensuration, que l'on voit migrer « tout naturellement $»$ du topos du professeur à celui des élèves (tdp.546 P : c'est quelque chose $\rightarrow \operatorname{tdp} .547 \mathrm{~d} 2$ : telle telle que si j'en prends $\rightarrow$ tdp.548 $\mathrm{P}:$ alors dis le bien chut c'est quelque chose telle que si $\rightarrow$ tdp.549 d2: j'en prends sept $\rightarrow$ tdp.550 P: oui vas-y $\rightarrow$ tdp.551 d2: sept j'en prend sept ça fait trois unités). La valence perlocutoire ${ }^{8}$ (Sensevy \& Quilio, 2008), dans cette succession de tours de parole, fonctionne sur le contrat didactique.

La transaction est envisagée dans tous ces aspects et pour toute la classe (tdp.554 P: je suis sûre que Caroline n'a pas entendu allez plus fort). Le tour de parole final de ce jeu d'apprentissage avec l'énoncé « et oui vous aviez oublié que la fraction ça a le sens que si j'en prends sept ça fait trois unités alors il a raison » porte à lui seul l'enjeu de toute la leçon, qui est ainsi déclaré non pas comme "synthèse » artificielle au terme d'une activité improductive mais au terme d'un travail ayant permis aux élèves d'éprouver par eux-mêmes un problème dans un milieu spécifique et l'outil conceptuel qui permet de produire des stratégies d'attaque efficace. L'ensemble du travail conjoint du professeur et des élèves a pu aboutir en fait à la production d'un contrat partagé et reconnaissable, qui a maintenant des chances raisonnables d'être utilisé comme arrière-plan commun, pour traiter ce type de problème dans ce type de milieu.

\section{Discussion}

La présence et l'intervention d'un chercheur / observateur auprès du «professeur d'essai ${ }^{9}$ ou directement avec les élèves vient sans doute limiter d'une certaine façon la portée des conclusions émises sur le travail du professeur, mais l'analyse du travail d'enseignement réalisé collectivement par les deux intervenants demeure. Cette leçon constitue donc un moment clé et rare pour entrer au cœur du dispositif d'enseignement « Rationnels et décimaux dans l'enseignement obligatoire » et pour saisir la subtilité de l'organisation et du fonctionnement des situations didactiques qui doivent être réalisées.

La description en jeux d'apprentissage montre la complexité de l'action d'enseignement et les difficultés rencontrées par le professeur dans la conduite de la séance. On retiendra d'une part le rôle décisif d'un énoncé particulier dans les différents jeux d'apprentissage et d'autre part un effet de reviviscence de la mémoire didactique indispensable à l'avancée du temps didactique. Nous revenons maintenant sur ces deux éléments 
saillants en centrant l'observation tout d'abord sur l'aspect langagier puis sur le plan de la mémoire didactique.

\section{La question de l'énoncé dense en savoir: "Quelque chose telle que si j'en prends..." :}

La description des jeux d'apprentissage montre le rôle central de l'énoncé « quelque chose tel que si j'en prends $[\ldots]$ ça fait $[\ldots]$ » à plusieurs niveaux. Si on s'attache au «fonctionnement » de cet énoncé au long de la séance, on remarquera qu'il joue tout d'abord le rôle de déclencheur de restitution d'un savoir institutionnel.

86. P : neuf fois plus grand alors qu'est ce qui quand j'en prends neuf est égal à onze comment ça s'écrit ça?

87. Elv/cl: en fraction

Cet énoncé rappelle une notion qui a l'épaisseur sémantique que l'on sait, il est donc décisif dans la reviviscence de la mémoire didactique qui permet aux élèves d'entrer dans les jeux d'apprentissage.

233. P: je vous en avais donné plein on avait mesuré des longueurs de baguettes

234. Elv/c4: ah oui sur la feuille il y en avait plein

235. P: voilà vous vous souvenez par exemple si je vous dis celle-ci là trois trois trois huitièmes trois pour huit la mesure trois pour huit si qu'est ce que ça serait si c'était une baguette

236. Elv /bl : c'est trois

237. P: alors oui elle est telle que si j'en prends

238. Elv /e 4: huit ça fait trois

239. P : ça fait trois unités ou bien si c'était les feuilles la feuille elle est épaisse telle que

240. Elv /e 4: si j'en prends huit ça fait trois millimètres (en cœur avec la classe)

L'usage de cet énoncé manifeste ici l'accommodation (Sensevy, 2011) de la connaissance au problème. Trois huitièmes, devenus trois pour huit, devient « si j'en prends huit, ça fait trois » et c'est aussi en références aux situations rencontrées dans les leçons initiales « huit baguettes ça fait trois unités » ou « huit feuilles de papier sont épaisses de trois millimètres ».

343. P : oui viens l'écrire viens l'écrire ici ah chut chut ah Guillaume il sait déjà écris moi là qu'est ce que ça signifie ça?

344. Elv /c4: un septième

345. P: un septième c'est quelque chose

346. Elv: /b2 si j'en prends
347. P: oui telle que si j'en prends sept ca fait une unité alors est ce qu'il lui est euh c'est ca qui qui l'a interpellée elle dit elle s'est dit je vais faire quelque chose avec ce sept là

L'accommodation de la connaissance est demandée par contrat: c'est ce que le professeur signifie ici.

567. P: c'est juste? // mais dites donc on le savait déjà rien qu'en le voyant écrit //rien qu'en le voyant écrit// trois septièmes trois pour sept qu'est-ce que c'est que trois pour sept 568. Elv /al : euh euh

569. $\mathrm{P}$ : c'est quelque chose oui

570. Elv/d2: telle telle que si j'en prends sept

571. P: alors dit le bien chut c'est quelque chose telle que si

572. Elv /d2: j'en prends sept // j'en prends sept euh sept euh sept euh

573. P: oui vas-y

574. Elv /d2: sept j'en prends ça fait trois unités

L'usage partagé de cet énoncé permet un travail de réexpérienciation de l'expérience première (Quilio, 2008; Sensevy, 2011).

106. P: neuf fois plus petit que onze vous êtes d'accord? oui donc si je prends dans l'autre sens onze c'est comment c'est comment par rapport à ca?

107. Elv/xx: neuf fois plus grand neuf fois plus grand (collectif)

108. P: neuf fois plus grand alors qu'est ce qui quand j'en prends neuf est égal à onze comment ça s'écrit ça?

109. Elv /cl : en fraction

Ainsi, jouer les jeux d'apprentissage nécessite, pour le professeur et les élèves, de pouvoir « parler » les fractions-mesure dans ces jeux. Le gain au jeu peut alors s'apprécier par le déplacement topogénétique de l'énoncé: . i l'énoncé parvient à « migrer» dans le territoire des élèves. Cet énoncé: «Quelque chose telle que si j'en prends...» est dense en savoir, et peut se définir dans ces usages avec un système sémiotique ou un système de notation comme un marqueur didactique, au sens de Culioli (Culioli, 2002).

«Un marqueur, si vous voulez, c'est une espèce de résumé, de concentré de procédures qui déclenchent et activent des représentations » (Ibid., p. 172).

[...]

Alors ce concept de marqueur est fondé sur l'idée suivante: nous avons affaire à une activité mentale, à laquelle nous n'avons pas accès directement mais uniquement par l'intermédiaire de ces marqueurs. 
Ce sont des opérations, à partir desquelles nous construisons des représentations, des catégories grammaticales, des mises en relation, de telle sorte que nous puissions référer, et que nous ayons entre nous un ajustement de nos systèmes de références, qui justement nous permettent de référer » (Ibid., p. 174).

Dans une telle perspective, les « énoncés denses en savoir " à condition qu'ils soient «joués " et « rejoués» en situation, peuvent peut-être être conçus comme des marqueurs didactiques et jouent un rôle majeur dans la création d'une référence partagée, car ils peuvent permettre en particulier d'ajuster les systèmes de références inscrits dans un système sémiotique.

\section{La question du phénomène de reviviscence de la mémoire didactique dans la réalisation de la transposition didactique:}

La mise en œuvre de cette leçon accorde une place centrale à la mémoire du système didactique et pour bien comprendre cette situation, il faut la penser comme un effet d'après-coup organisé. Je reprends ici l'idée fondamentale, dans le travail de Centeno, d'historicité du savoir, qu'on peut trouver par exemple dans le théorème suivant de sa thèse:
«Plus les événements sont déterminés par une didactique du maître et par une épistémologie de l'élève précise et commune à tous, moins ils sont susceptibles de réorganisation d'après-coup et d'ajustement significatifs, personnels, adaptés au vécu, et donc, moins ils ont de sens »(Centeno, 1995, p. 173).

On comprend bien, dans les jeux d'apprentissage que je viens de décrire, que le déroulement de la séance nécessite un travail très particulier du professeur. Il s'agit de faire en sorte que l'élève puisse actualiser (aux deux sens du terme) un milieu ancien dans une situation nouvelle. Ce travail mésogénétique peut fonctionner parce que les situations sont structurantes de l'expérience des élèves, et qu'elles peuvent être réexpériencées à partir des marqueurs précédemment associés au système sémiotique actif dans la situation. Sous cette description, on peut voir se dessiner une forme particulière de l'étude, un « temps des situations » (Sensevy, 2004; Quilio, 2008; Sensevy, 2011) où le mode de transposition didactique que le professeur doit réaliser induit une temporalité de l'étude différente de celle induite par la présentation, dans un cours professé, d'un texte du savoir déjà écrit (Chevallard, 1985). L'avancée du temps didactique ne repose pas ici sur l'introduction par le professeur d'objets nouveaux, mais sur la mise en perspective d'une situation initiale fondatrice par la production d'une référence évolutive. 


\section{NOTES}

1. Une étude plus détaillée de certains aspects de l’ingénierie a été produite dans ma thèse (Quilio, 2008) . Des éléments de cette étude ont été repris et prolongés par G. Sensevy (Sensevy, 2011).

2. Je me réfère au concept de « voir » du chapitre XI des Recherches philosophiques (Wittgenstein \& Collectif, 2005) et du travail d'explicitation de Laugier sur ce texte (Laugier \& Chauviré, 2006).

3. Ce tableau est issu de Quilio 2008 et a été partiellement repris et modifié par G. Sensevy (Sensevy, 2011).

4. Une fraction s'appréhende alors par commensuration dont une définition mathématique est la suivante: une quantité (si elle existe) sera les a/b d'un entier si en le reportant b fois (en en prenant b identiques à ellemême) on obtient a entiers.

5. Sous cette expression, je décris la dynamique du partage des actions mésogénétiques ou chronogénétiques des transactants et plus précisément ici le fait que le professeur marque un partage du milieu avec ses élèves.

6. Le mot réticence est envisagé dans son sens, vieilli en français, d'une « omission volontaire de ce qui pourrait ou devrait être dit », et toujours actif dans l'anglais « reticent ».

7. Dans la dynamique de la mésogenèse, c'est le professeur qui décide ici de l'action que les élèves ont à conduire dans le milieu.

8. Cette notion de valence perlocutoire des énoncés professoraux est une spécificité des interactions langagières en classe (Quilio, 2008). Cette spécificité pourrait se décrire comme un pouvoir de certains énoncés à faire agir les élèves de façon idoine.

9. J'emprunte ici cette formule à Alain Mercier

\section{RÉFÉRENCES}

Brousseau, G. (1981). Problèmes de didactique des décimaux. Recherches en didactique des mathématiques, 2 (3), p. 37-127.

Brousseau, G., \& Brousseau, N. (1987). Rationnels et Décimaux dans la scolarité obligatoire. Université de Bordeaux 1.

Brousseau, N. (1987). La mesure en cours moyen $1^{\text {re }}$ année. Compte rendu d'activités. Université de Bordeaux 1 IREM de Bordeaux.

Centeno, J. (1995). La mémoire didactique de l'enseignant (Thèse posthume inachevée). Bordeaux 1.

Chevallard, Y. (1985). La transposition didactique (Grenoble, La Pensée Sauvage). Rééd. augmentée (1991).

Culioli, A. (2002). Variations sur la linguistique. Entretiens avec Frédéric Fau. Klincksieck.

Laugier, S., \& Chauviré, C. (2006). Lire les » Recherches philosophiques». Vrin. 
Quilio, S. (2008). Contribution à la pragmatique didactique. Une étude de cas dans l'enseignement des nombres rationnels et Décimaux à l'école primaire. Université Aix-Marseille 1.

Ratsimba-Rajohn, H. (1992). Contribution à l'étude de la hiérarchie implicative. Application à l'analyse de la gestion didactique des phénomènes d'ostension et de contradiction. Rennes 1.

Sensevy, G. (2004). Quelques remarques à propos de la notion d'étude. Exposé au DAEST, Bordeaux, 6 décembre 2004.

Sensevy, G. (2011). Le Sens du Savoir Éléments pour une Théorie de l'Action Conjointe en Didactique. De Boeck.

Sensevy, G., \& Mercier, A. (2007). Agir ensemble: L'action didactique conjointe du professeur et des élèves. Presses universitaires de Rennes.

Sensevy, G., \& Quilio, S. (2002). Les discours du professeur. Vers une pragmatique didactique. Revue française de pédagogie, 141 (1), p. 47-56.

Vernant, D. (1997). Du discours à l'action. Presses universitaires de France - PUF.

Wittgenstein, L., \& Collectif. (2005). Recherches philosophiques. Éditions Gallimard. 